\title{
VIII.-PRIMARY AND SECONDARY QUALITIES.
}

$B y$ G. F. Stout.

"Everyone," says Thomas Reid, "knows that extension, divisibility, figure, motion, solidity, hardness, softness, and fluidity were by Mr. Locke called primary qualities of body; and that sound, colour, taste, smell, and heat or cold were called secondary qualities. Is there a just foundation for this distinction? Is there auything common to the primary which belongs not to the secondary? And what is it?"

The first of the questions thus formulated by Reid may be regarded as settled. No competent person doubts that the distinction has a real foundation. But on the second question it seems to me that there is much room for discussion. The answer which is most familiar and also most precise and clear is, I think, definitely wrong. On the other hand, those philosophers who reject this false doctrine do not in general substitute for it any positive and detailed view of their own which I find myself able to accept. They are more bent on showing their opponents do not solve the problem than on solving it themselves.

The definite view commonly accepted by representatives of Physics and Physiology and also by many Philosophers is that the primary qualitics really do belong to bodies, whereas the so-called secondary qualities are not qualities of external things at all but qualities of sensations experienced by percipient. minds when external things stimulate the organs of sense in certain ways.

Postponing consideration of that part of this theory which relates to primary attributes, let us first examine the contention that the secondary attributes are attributes not of bodies but of sensations. This contention is urged against 
what is presumed to be a natural fallacy of ordinary thinking. It is directed against a supposed confusion of common sense between qualities of sensation and properties of external things. When the plain man says that burning coal is hot he is taken to mean that the peculiar quality of the sensation felt by him when he holds his hands near the fire is actually part of the nature of the burning coal. Similarly, when he says that grass is green he is taken to mean that the specific quality of the visual appearance of the grass when he looks at it by ordinary daylight inheres in the grass itself. Against such a view it is urged that heat in this sense is no more in the fire, and that greenness in this sense is no more in the grass than pain is in the surgeon's knife. Grass and burning coal being insentient cannot experience temperature-sensations or colour-sensations. Hence, all that the plain man ought to mean in asserting, for example; that the fire in the grate is hot, is that it has a power of making him feel hot under certain conditions. If we enquire on what this power depends we are referred to the primary properties of the fire, such as the vibrations of its molecules.

Now, this criticism of common sense seems to me to be founded on a misconception of the actual procedure of ordinary thinking. In principle the plain man is not really guilty of the confusion with which he is charged. He does not in general confound intrinsic characters of his own sensations with attributes of external things. On the other hand, I admit and maintain that in ascribing secondary qualities to corporeal things he does not merely mean their power to produce certain sensations in us. His point of view is not that of his critics; but neither is it that which his critics ascribe to him. What it really is remains to be investigated. When it is fairly presented it will, I think, be found defensible, and indeed the only one which is defensible, for the case of primary as well as of secondary qualities.

In investigating the view of the plain man, the very worst course we can pursue is to ask the plain man himself what he 
means. His thought is through and through based on latent assumptions and implicit inferences which he does not ordinarily attempt to define and formulate. So soon as he attempts to define and formulate them he becomes a philosopher. But his first untrained ventures in philosophical analyses are sure to be extremely crude and unsatisfactory. To depend on his raw opinions about such topics as we are here concerned with is like depending on a child for an account of the psychology of his own mental processes. If we wish really to understand common sense we must follow and analyse its actual procedure with the view of bringing out the presuppositions which underlie this procedure. We must not ask it directly to give an account of these presuppositions.

This being understood, we may now proceed to enquire what is meant in ordinary, unreflective thought, when things are asserted to be hot, cold, sweet, red, blue, \&c. We may take as typical the case of temperature. I touch a piece of iron and feel a sensation of heat. I consequently assert that the iron is hot. What do I mean by this assertion? Not merely that the body is actually producing a certain sensation in me. For I presume that the iron was hot before I touched it or came near it, and that it will continue to be so if I remore my hand and go away. Do I, then, regard the hotness of the iron as a power to produce a sensation of heat under certain assignable conditions? That is certainly a part of my meaning. But it is far from being the whole of it. On the contrury, the hotness of the iron is thought of as being a quality in it as specific and positive as the quality of my sensation when I touch it. The one is no more a mere possibility than the other. This is shown by the fact that in ascribing secondary qualities to things we normally think of the things as if they were actually producing the sensations in a hypothetical percipient. When we think of iron as hot and gradually cooling down, we think of it as if it were in fact generating gradually diminishing sensations of heat in some one near enough to 
feel them, even though we are quite well aware that no one is actually present. When we now think of the books in our library as red, blue, green, and yellow, we think of them as they would appear to us were we there to look at them in ordinary daylight. But we proceed quite otherwise in the case of mere possibilities. When we think of a hayrick as inflammable we do not do so by representing it as if it were actually in flames. When we think of a window pane as brittle we do not represent it as actually being broken. In such case we consciously distinguish between possibility and its actualisation. We do not lose sight of the possibility as such and mentally substitute the actuality.

Are we then to conclude that common sense actually supposes sensatious exist and change when no one actually experiences them? Before charging it with so flagrant an absurdity we ought, I think, to enquire first whether there may not be another and more defensible interpretation of its procedure. It seems to me that there is another which is not. merely defensible, but the only one which is defensible.

There are two main points to be emphasised. The first is that the sensations which mediate our knowledge of the secondary qualities do so only in so far as they represent, express, or stand for something other than themselves; and their representative function being independent of their actual existence at this or that moment in this or that unind, they may be validly thought of as if they existed when in fact they do not and cannot exist The second point is that the distinction between what is represented and its sense-representation is only a latent presupposition of ordinary thinking. The plain man does not in general formulate it, though in our logical analysis of his procedure we must formulate it for him. What are called the secondary qualities of matter are not identified with what is represented in distinction from its sense-representation, nor yet with the sense-representation in distinction from what it stands for. It is rather the 
complex unity former by both together and commonly left unanalysed.

The representative function of sensation may be best exhibited by contrast with cases in which it is absent.

The gradual diminution and final discontinuance of heat sensations which ensue when the sentient organism is withdrawn from the perceived object is without representative value. It depends on movements of the organism which neither produce nor imply any relevant change in the object. Hence the concomitant change of sensation is rightly regarded as merely a change in the sensible appearance of the thing which does not express or represent any corresponding alteration in the thing itself. All such variations in sense experience may therefore be ignored or cancelled as irrelevant in mentally dealing with external objects. Now, if it is legitimate to cancel out variations of sensible appearance due merely to variable conditions of perception, it is for that very reason legitimate to represent the object as it would appear under uniform conditions of perception, whether or not it is actually so perceived, or, indeed, whether or not it is perceived at all. All sensible changes and differences under uniform conditions of perception express or represent corresponding changes in things perceived; for by hypothesis they can be due to no other cause (and the principle of causality underlies the whole procedure). Hence we are interested in their representative value, and not in their actual existence. We may and do think of them as if they actually existed when they do not actually exist. We may, for instance, legitimately represent the sun as sensibly hot before any sentient beings appeared on this planet. Such procedure is logically justifiable provided always that one grand rule is observed. In comparing one thing with another, or different states of the same thing, it is always presupposed that the conditions of perception are sufficiently similar to prevent confusion between mere difference of sensible appearance and difference in the nature of the things 
compared. The uniformity is, of course not absolute, but only sulficient in view of the fineness of discrimination required.

Coming now to our second point, we must insist that, though this distinction between the independent nature of material things and the mode in which their nature is sensibly represented is logically presupposed in ordinary thinking, yet it is not, as a rule, explicitly recognised. On the contrary, both the sensible representation and what is represented are equally included in what are called the secondary properties of matter. Were it otherwise the secondary properties would be for common sense mere powers, or occult qualities, or if they had a positive and specific content, this would be definable only in terms of the primary properties. In fact, however, they have for common sense a positive and specific qualitative content of their own. They possess this content because the qualities and relations of temperature, colour, sound, smell, and taste sensations enter in virtue of their representative function into the essential constitution of the corresponding secondary attributes of matter.

If this analysis is correct it follows that the secondary attributes of matter are correlated but not identical with corresponding qualities of sensation. Hence, in ordinary language we speak not of a yellow sensation or a hot sensation, but of a sensation of yellow or a sensation of heat. In ordinary life our predominant interest is in the sensible properties of bodies with which we are conversant through the medium of sense, and therefore we name these directly. The corresponding sensations are named indirectly by reference to these. But it would be a gross error to suppose on this account that the sensations as such are without qualitative content. On the contrary, whatever qualitative content belongs to the secondary properties of matter presupposes and is derived from the qualitative content of sensations qud sensations. This is to be borne in mind when we come to deal with primary qualities. 
We have seen that in comparing objects with each other as regards their secondary qualities, standard conditions of perception are presupposed. The selection of the standard conditions is, of course, in part determined by convenience of relerence. But there is also another motive which involves an important principle. Distinct sensible appearances are preferred to those which are relatively indistinct. The principle involved is that difference in the sensible appearance under uniform conditions of perception always expresses difference in the things perceived, whereas absence of difference in the sensible appearance does not necessarily express absence of difference in the things perceived. In the latter case all that is implied is that the differences which fail to appear are slighter than those which do appear. For this reason a near view of an object is preferred to a more distant view, and in determining the proper or constant colours of things we think of them as they appear by ordinary daylight and not as they appear in the dusk when only different shades of grey are discernible and other colour distinctions are hidden from view.

This analysis of the secondary attributes of matter holds good in all essential respects for the primary also. The primary, like the secondary, are correlated but not identical with intrinsic characters of sensation, especially visual, tactual, and motor sensation. The correlation is essentially of the same kind for both. Sensation enters into the constitution of the primary attributes only in so far as certain features of sense-experience represent something other than themselves, and it is only because this representative function is logically independent of the actual occurrence and fluctuation of senseaffections that the primary qualities can be validly thought of as existing in the absence of percipients. We are justified in thinking of matter as extended and movable in space before the existence of sentient being. But we have exactly the same justification for thinking of it as hot or coloured. Finally, the positive and specific nature of the primary 
qualities no less than that of the secondary is derived from corresponding sensations.

We may take as our chief example the most fundamental of the primary qualities-Extension. In ordinary language it seems strange to speak of sensations as extended. The reason is that they are not extended in the same sense as corporeal things. Bodies are extended in space. But touch and sight sensations do not in the ordinary sense of the words occupy Space. They do not occupy any part of the single, homogeneous, infinite space which embraces all material things and their distances. They do not occupy any part of the space in which Cardiff or Oxford is so many miles from London, and in which bodies attract each other inversely as the square of their distance. None the less, touch and sight sensations have an intrinsic character correlated with spatial size and shape, just as the quality of sensations of yellow is correlated with the yellowness of buttercups and oranges. We may call this intrinsic character sensible extension. Since in ordinary life we are interested in sensible extension mainly as an expression or manifestation of spatial extension, spatial extension may be called real and sensible extension apparent. Thus we contrast the apparent size of a thing as seen at this or that distance from the eye with its real size as measured in feet or inches. Spatial or real extension is throughout homogeneous; sensible extension is of two kinds, the visual and the tactual. Their difference is perhaps comparable to that of the intensity of light sensations and the intensity of sound sensations.

Consider first visual extension. On closing the eyes though we cease to see external objects or any part of our own bodies, there is still a field or expanse of visual sensation which may be entirely grey or variegated with colour. Each distinguishable part of this field or expanse has local relations of position and distance to other parts, and the whole is a single continuous extensive quantum. Yet the visual expanse thus 
presented for our attentive scrutiny does not occupy any part of space. If it is in space it must be here or there. But we cannot from the nature of the case say where it is. There is no room for it in the space occupied by bodies. It may be suggested that the extent of the visual field coincides with that of the retina. To this it seems a sufficient reply that the extent of the visual field or its parts is not merely dependent on the size of the retina or its parts, but also on its anatomical structure-on the packing of rods and cones. Thus, the same stimulation of the lateral portions of the retina gives a less extensive sensation than stimulation of the foven centralis. Again, if the expanse of visual sensation occupies any portion of space it must be conterminous with other outlying portions of space. But in this sense it is boundless though not of course infinite in magnitude. Parts within it are bounded by other contiguous parts, but in its totality it does not form part of a more extensive whole, and it has therefore no limits which are in any sense spatial. It has no shape. If you doubt this try to discover what its shape is. In the next place, if it occupied space, it would be commensurable with other spatial quanta. It ought to be possible to express its magnitude in feet or inches. But this is an intrinsic impossibility. We cannot, for instance, say that it is equal in extent to the total tract of the external world which comes within the range of vision when the eyes are open. For what we can thus embrace in one view may vary indefinitely in extent. It may include the expanse of the starry heavens or it may be confined to the walls of a room. Again, a part of space may be conceivably empty; but the conception of a vacuum has no application to visual extension. There is no visual extension where there are no colour and brightness sensations.

Finally, space is a common object, in principle equally accessible to all of us. But each of us is directly acquainted only with his own visual tield. The exteusion of colour and brightness sensations disappears with the sensations themselves, 
when a man dies or is afflicted with cortical blindness. But no part of space is thereby annihilated.

What has been said of the visual field of the closed eye holds also in all essentials for the visual field of the open eye. To avoid tedjous repetition, I shall leave you to make the application for yourselves. I now pass on to consider the relation of real or spatial extension to the visual extension, which is its sensible appearance. The relevant facts are familiar to everyone. Everyone knows that the size and shape of the visible appearance of a thing vary indefinitely as we approach or recede from it, or otherwise change our position in relation to it, while the thing remains constant in shape and size. The visual appearance of a match-box in my hand may be co-extensive with the visual apperance of a distant mountain. The entire disappearance of things when we go far enough away is a limiting case of such variations. Now, we cannot identify the real size of a thing with the whole series of possible changes in the extent of its visible appearance, nor yet with the fixed order of their possible occurrence. For the real extension may remain constant, while its appearance alters, and it does not in its own nature include or imply the concept of change. Still less can we select this or that apparent extension and identify it with the real. For each of them has in principle just as much and just as little logical title to be so regarded as any of the others. They pass into one another by continuous gradations, so that it is impossible to fix on one only, to the exclusion of all others differing ever so slightly from it. We do, indeed, usually think of the real extension in terms of its visual appearance under certain conditions. But these conditions are loosely determined, and they are variable according to our convenience or the degree of accuracy required. We may choose any conditions we please, provided we abide by them with sufficient strictness in comparin $_{\tilde{\xi}}$ one object with another, and provided they yield visual appearances sufficiently distinct in view of the required fineness 
of discrimination. If we are interested in differences too slight to be revealed to the naked eye, we have recourse to a magnifying glass; and the visible extent of the thing under the magnifying glass has just as much claim to be identified with its real magnitude as its extent when it is seen by the naked eye.

In general, extension as a characteristic of visual sensation is quite distinct from the extension of things in space. And yet if we leave tactual experience ont of count, extension as a property of bodies and the space in which bodies are extended derive their positive and distinctive content from the extensiveness of visual sensation. Real extension is, indeed, something other than visual extension; but its extensive character belongs to it only inasmuch as this something is represented in terms of the extensiveness of sight sensations or of touch sensations.

Since Berkeley's time it has been customary to assume that touch affords an immediate revelation of the real size and shape which is denied to sight. Visual extension is admitted to have a merely representative value, but the reality represented is identified with tactual extension. It is easy to show that this distinction is indefensible. Apply the blunt end of a pencil to the forehead, to the lips, to the back of the hand, to the tip of a finger, to the drum of the ear. The resulting tactual sensations vary conspicuously in extent, though the areas of the skin affected are throughout equal and the surface with which they are brought in contact remains constant in size. None of the tactual extensions has any better logical claim than the others to be identified with the real extent either of the skin stimulated or of the surface applied to it; and their rival claims are mutually destructive. Skin sensibility is also variable in this respect from one individual to another; it is different in the child and the adult; it is affected by diserses of the brain, and by the use of drugs such as narcotics. 
The case is not altered if we turn to what are called kinæsthetic sensations-muscle, joint, and tendon sensations. Berkeley identified the real distance between one body and another with the series of joint, muscle, and tendon sensations which would be experienced in moving from one to the other. We ask what series is meant? Is it that which would be experienced in walking, or in running, or in hopping on one leg? Is it that which would be experienced by a child of three or by an adult with a long stride? Which of these series consists of feet and of yards? There is ouly one tenable answer to such questions, whatever sense-experience we may be considering. Given uniform conditions of perception, whatever these may be, differences of sensible extension and differences of more and less in the series of motor sensations represent differences in the external world and the differences as thus represented-the differences together with the mode of representing them are what we call differences of real, physical, or spatial extension. The more differences are discernible in the sensible appearance under sufficiently uniform conditions, the fuller and more exact is our knowledge of real size, shape, and distance. But we cannot fix on any set of conditions and identify the corresponding sensible extension with extension in space. Neither tactual nor visual extension occupy any part of the space in which bodies attract each other inversely as the square of the distance.

It follows that the single infinite all embracing Space of Kant is not, as he assumes, a form of sensibility at all. It is essentially a form of what he calls external objects, and as such already presupposes the work which he ascribes to the categories. Yet it is presented by him at the outset as a pre-condition of the work of the categories, and is from the outset confounded with sensible extension. No wonder that he found in it a most convenient middle term between the pure concepts of the understanding and the disparate matter of sense. This confusion, and a similar one relating to time, seem to me to vitiate 
the argument of Critique of Pure Reason from beginning to end.

It is needless for me to deal separately with the other primary qualities, resistance and mobility. These are correlated with sensations of muscular tension and with sensible displacement within the field of sight and touch just as real extension is correlated with tactual and visual extension.

Our general result up to this point is that there is no essential difference between the primary and secondary attributes of matter so far as regards their connexion with sense-experience. Both are in one way independent of sense and in another dependent on it. Both are dependent on it for the positive content which makes them more than mere powers or occult qualities. Both are in the sense explained independent of it as regards their existence.

What, then, is the true foundation of the undoubted distinction between then which is marked by calling them respectively primary and secondary qualities? So far as I can see the difference lies in their respective relation to the interaction of material things. The executive order of the material world can be expressed only in terms of the primary and not in terms of the secondary properties of natter. The unity and continuity of material processes is intelligible only through the unity and continuity of Space. The system of uniformities of co-existence and sequence, and of quantative equivalences and correspondences which constitutes the order of physical nature in its causal aspect can be formulated only in terms of extension, motion, and tension. On the other hand, we find no such constancy, continuity, and quantitative equivalence in the occurrence of sounds, colours, or smells. There is, e.g., no system of laws according to which sounds succeed each other or vary concomitantly in loudness. But you can always obtain a certain note by striking the right key of a piano, and by striking more or less hard you can make it louder or softer. 
This is mere common-place and needs no further exposition. But something must be said concerning the implications of this common-place. For an attempt may be made to use it so as to upset the results of our previous analysis. It may be urged that if agency belongs to matter in virtue of its primary properties, these properties must have an existence independent of sense-experience such as does not belong to the secondary. The steam hammer beats out the bar of steel and the sun attracts the planets independently of anyone's sensations. The primary properties are presupposed in the processes by which the organs of sense are stimulnted; how, then, can they be dependent on the resulting sensations? Again, science finds itself bound to postulate operative conditions and therefore primary qualities where the secondary can have no place. Thus the particles of luminiferous ether cannot themselves be coloured. Does not this point to a radical difference between the primary and the secondary qualities in their relation to sense-experience?

In reply to such contentions, I need only refer again to the distinction between sensible representation and that which is represented. What is represented exists and operates independently of the coming and going of the sensuous presentations through which we express its existence and operation. It is independent of these as the topography of England is independent of the map of England, or as the rise and fall of temperature is independent of the rise and fall of the mercury in a thermometer. There is a systematic agency which we express in terms of sensible extension, motion, and muscular tension; so expressed it is what we call material causationthe interaction of bodies in space. But the features of senseexperience through which we represent it contribute nothing to its agency. On the other hand, since the representative value of sense-experience is independent of the existence of sensations, we may validly represent in terms of sensible extension, motion, and resistance, the processes through which 
these and other sense-experiences come to be and cease to be. From the same point of view, it is easy to account for the existence of- primary qualities in the absence of the secondary. There is no reason why sensible extension, motion, and resistance should not have a representative value, where sensible colour, sound, heat, and taste have none, just as relative positions and distance or a map have representative value where the flatness of the map, its absolute size, and the colouring of the counties have none. Similarly, in thinking of empty space, representative value attaches only to sensible extension and the series of muscle, joint, and tendon sensations.

And now I might regard my immediate problem as disposed of, so far as I am able to deal with it, were it not that a rival theory still demands attention - the theory which resolves the material world into a system of possibilities of sensation. This view is advocated in its purity by Mill, and with a well known reservation by Berkeley, and Kant at times seems to lean to the same side. According to it sensations have indeed a representative valuc, but what they represent is always only the possibility of getting other sensations in a fixed and systematic order. The material world is supposed by it to be constituted of actual sense-experiences, together with the systematic order of possible sense-experience. Against this doctrine I urge in the first place that the order of possible sensations is widely divergent from the order of the physical world and its processes. Consider the fluctuation of the visible appearance of a body as we approach or recede from it, and the variations of tactual extent as a body is applied to different parts of the skin. Such differences are not differences in the size of the body itself, and they are not included in what we mean when we say, for instance, that the body is three inches long. Again, as Kant has insisted, there is a contrast between the succession of our sensations and co-existence in the external world. The back and front of a house co-exist, but the corresponding sensations are successive. Finally, how can the 
internal content of a solid body be resolved into any possible series of sensory presentations. Slice it as you will you only disclose surfaces; not solid content, but only the boundaries of solid content. The supporters of the theory usually meet such arguments by the help of extravagant illustrative hypotheses. They urge, for instance, that sentient beings, otherwise conditioned than ourselves, would experience simultaneously the sensations which we can experience only successively. But the appeal to such an imaginary percipient implies that at least the successive order of our own sensations, in spite of its fixed and systematic character, forms no part of the order of the physical world. Nor can the theory so long as it remains self-consistent supply us with any reason why the imaginary experience should be preferred to ours. The relevant difference cannot lie in the diverse conditions of perception. For these conditions, according to the theory, can themselves consist only in an order of actual and possible sensations. There seems to be no assignable ground for preferring the fictitious experience unless we already presuppose a knowledge that, e.g., the order of the external world is co-existent as contrasted with the successive order of its sensible appearance to us. There is a still more fundamental objection to the doctrine. It dislocates and transposes the relation of the possible and actual. It commits the old blunder of dogmatic metaphysics, making essence prior to existence, investing it with a pseudo-existence, and deriving actual existence from it. Possibility essentially presupposes actuality. To say that something is possible is to say that there is something actual which would behave in a certain way under certain conditions. But the doctrine we are discussing: deals freely in mere possibilities without any such relation to anything actual; these naked possibilities it regards as the source of actual sensations, and to intensify the absurdity it supposes that actual changes take place in these naked possibilities, and also that change in one naked possibility determines changes in others. Take Mill's example of the table 
which is believed to exist when no one is present to perceive it. This belief is construed as meaning that if any one went into the room, or were now in it, and suitably directing his organs of sense he would be aware of certain sensations, e.g., of a group of visual sensations. Now, the going into the room and the being in the room and the adjustment of the sense organs must, of course, in accurdance with the theory be simply identified with having certain possible sensations in a certain order. Suppose these sensations actualised. It does not therefore follow that a table becomes visible. I should have just the same sensations without seeing any table if no table were there. The table itself is that which so reacts, or would so react under the assigned conditions, as to give rise to those actual sensations which are called the visible appearance of the table. But according to the theory under discussion the table is nothing actual but only a naked possibility. Thus a naked possibility is supposed to operate as an agent giving rise to something actual-to actual sensations. To crown the absurdity, it is supposed to effect this by determining other naked and unactualised possibilities of sensation which again consist, in changes taking place in yet other naked and unactualised possibilities. For such is the only interpretation which the theory can put on the proposition that the table affects the sentient organisin by reflecting light to the eye and so setting up molecular processes in the nervous system.

For these, among other reasons, I feel bound to reject the doctrine of Mill and Berkeley, though I imagine it is held in substance by some at the present day who belong to a very different school of thought. I am quite prepared to be told by thinkers with whom I have at bottom much in common that my own position is at least as untenable. I expect to be charged with reviving the exploded doctrine of things in themselves, disparate and discontinuous with our immediate experience. With a clear conscience I plead not guilty to all counts of this indictment. 
There is, indeed, a sense in which I postulate things in themselves. But in this sense I do not see how anyone can deny them. I postulate things in themselves in the sense in which another man's toothache is relatively to me a thing in itself as having an inner being of its own which $I$ do not immediately experience though I may know of it. I postulate them in the sense in which my own past toothache is a thing in itself relatively to my present existence inasmuch as I do not immediately experience it when I remember it. But so understood things in themselves are surely admitted facts and not exploded figments of an obsolete metaphysics.

In distinguishing between sensible representation and what it represents I do not commit myself to any irreducible dualism. I do not divide the universe into disparste and discontinuous parts. On the contrary, the existences and processes which have an inner being of their own are the very same existences and processes which as sensibly represented constitute the world of material phenomena. It is with the things in themselves, if we choose to name them thus, that we are incessantly conversnnt through the medium of sense. They constitute the constant presupposition and necessary complement of our conscious experience. Their inner being cannot therefore be disparate and discontinuous with our own conscious life. On the contraly, we and they must form part of one continuous whole. They must be bone of our bone and flesh of our flesh. This neans for me that their inner being is ultimately psychical. Indeed, like Lotze, I fail to understand what "inner being" can possibly mean unless it means "psychical being."

But how, it wiil be asked, can we know all this? Am I not, begging the question in assuming that in any relevant sense we are or can be conversant with the things in themselves so as to be able to represent them in terms of sensation? Granted that they determine modifications of our sense-experience how can we be awure of anything except the resulting sensation. The scratch, as H. Stirling says, knows nothing of the thorn. 
Confined at the outset to our own states-our own immediate experience-by what possibility can we ever transcend these? Evidently we can only do so by way of inference. But how can we infer from $A$ to $B$, when $B$ is supposed to be something with which we are totally unacquainted?

As regards this last question I would point out that unless what is inferred is other than the datum there is no inference. All that is necessary for inference is (1) that the datum shall be by its intrinsic nature a fragment of a wider whole, and shall therefore point beyond itself to its own necessary complement; (2) that there shall be a thinking and willing being capable of discerning and actively eliciting the implication. Turning to our special problem, I admit that on my view the primary datum for the individual mind is its own immediate experience. But this proposition seems to me to be not only distinct from but in direct contradiction to the statement that in the first instance we know only our own states. If our own states could be known in pure isolation from aught else, they would not be data. An isolated datum is a contradiction in terms. $\boldsymbol{A}$ datum is a datum only because being essentially a fragment it points beyond itself; and what it thus implies cannot be merely being in general or merely the absolute, but always something as specitic as itself. A state of feeling incapable of revealing anything beyond itself that would be a petty absolute. This applies to the primary datum-immediate experience; the immediate experience of each of us being a fragment of the one continuous universe must manifest itself as such to a thinking being. Immediate experience must from the outset be inseparably blended with immediate inference, and this in manifold ways. It is in this direction and not in any a priori contribution of the understanding that I would look for the source and the justification of the Kantian categories.

I can here only say one word or two to indicate the bearing of these general remarks on the question, how we can know the 
existence and processes which, as represented in terms of sensation, constitute the external world. The only answer which I have to offer is an old one, but one which has not, so far as I can discover, been yet properly stated or understood or intelligently criticised. I turn for a solution to the intrinsic nature of conation and will and the mode in which conation and will find themselves conditioned as regards success or failure in the control of sense-experience. From the same source I coincidently derive the concept of tendency which seems to be the most distinctive and indispensable element in concrete causality. It lies beyond the limits of this paper to follow out this line of thought in detail. It is sufficient for my present purpose if I have succeeded in showing how I conceive the problem without attempting to solve it. 